\title{
Title: Non-invasive brain stimulation and non-invasive peripheral stimulation for neglect syndrome following Acquired Brain injury
}

Running Title: Non-invasive brain and peripheral stimulation for the treatment of neglect

Giuseppe Lucente ${ }^{\mathrm{a}, \mathrm{b}, \mathrm{c}, \mathrm{d}}$; Josep Valls-Sole ${ }^{\mathrm{e}}$; Narda Murillo ${ }^{\mathrm{c}, \mathrm{d}, \mathrm{f}}$; John Rothwell ${ }^{\text {; Jaume }}$ Coll a,b,c,d; Antoni Davalos ${ }^{\mathrm{a}, \mathrm{c}, \mathrm{d}}$; Hatice Kumru c, e,f;

a Department of Neuroscience, Hospital Germans Trias i Pujol, Badalona, Spain

b Grup de Recerca en Malalties Neuromusculars i Neuropediatriques, Department of Neurosciences, Institut d'Investigació en Ciències de la Salut Germans Trias i Pujol, Universitat Autònoma de Barcelona, Badalona, Spain

c Universitat Autonoma de Barcelona, 08193 Bellaterra (Cerdanyola del Vallès), Spain

d Fundació Institut d'Investigació en Ciències de la Salut Germans Trias i Pujol, Badalona, Spain.

e EMG department, Hospital Clinic, Barcelona

fFundación Institut Guttmann, Institut Universitari de Neurorehabilitació adscrit a la UAB, 08916 Badalona, Spain.

g Sobell Department of Motor Neuroscience and Movement Disorders, Institute of Neurology, Queen Square, University College London, London,UK

Conflict of interest: None to declare

Contributorship:

GL, JR and HK designed the study and GL, NM and HK reviewed de literature; JVS,JR AD and JC revised the manuscript and corrected the proof.

All authors reviewed and edited the manuscript and approved the final version of the manuscript.

Acknowledgements: None

* Correspondence to:

Hatice Kumru, MD, PhD.

Hospital de Neurorehabilitació Institut Guttmann

Camí Can Ruti s/n. Barcelona 08916 Barcelona, Spain.

Tel. + 349349777 00; Fax +34 934977715

Email: hkumru@guttmann.com 


\section{Abstract}

Background: Hemispatial neglect is a frequent condition usually following non-dominant hemispheric brain injury. It strongly affects rehabilitation strategies and everyday life activities. It is associated with behavioural and cognitive disability with a strong impact on patient's life.

Methods: We reviewed the published literature on the use of noninvasive brain stimulation, including repetitive transcranial magnetic stimulation (rTMS) and transcranial direct current stimulation (tDCS), and of non-invasive peripheral muscle stimulation, as therapeutic strategies for rehabilitation of neglect after acquired brain injury (ABI), such as in stroke or in traumatic injuries. The studies were grouped as controlled or uncontrolled studies in each stimulation technics.

Results: Thirty-four studies were identified and 16 on rTMS, 10 on tDCS and 8 on vibration. All studies were conducted in adult patients who suffered a stroke, except for one that was conducted in a patient suffering traumatic $A B I$ and another that was conducted in a patient with brain tumour. In spite of significant variability in treatment protocols, patients' features and assessment of neglect, improvement was reported in almost all studies with no side effects.

Conclusion: Non-invasive brain stimulation and neuromuscular vibration are promising therapeutic neuromodulatory approaches for treatment of neglect. Further randomized-controlled studies are needed to corroborate their effectiveness as separate and combined techniques.

Keywords: Non-invasive-brain-stimulation; Neglect; Neuromuscular Vibration; Rehabilitation; Stroke; Acquired brain injury; 


\section{Introduction}

Visuospatial neglect is a common and disabling condition following unilateral brain damage, especially right hemispheric stroke. ${ }^{1}$ Its clinical presentation is characterized by unawareness of the side contralateral to the hemispheric lesion, with a strong attention shift to the ipsilateral side.

The clinical picture of neglect is variable, ranging from mild to profound degree of impairment. In severe cases, patients may be unaware of the entire hemispace and show inability to use their contralesional limbs even though they have little or no weakness (motor neglect). ${ }^{2,3}$ Importantly, neglect may be associated with anosognosia, denying the existence of the impairment. ${ }^{3-5}$

The pathophysiological bases of neglect are probably heterogeneous, as different lesion sites have been associated with the clinical picture. ${ }^{4,6-10}$ The syndrome is much more likely to occur with involvement of the right than of the left hemispheres. ${ }^{1,11}$ Classical studies from Vallar and Husain 5,12 identified the right inferior parietal lobe (IPL) and temporoparietal junction (TPJ) as the most likely anatomical substrates of neglect. More recently, other cortical areas such as the inferior frontal lobe ${ }^{13}$ and the superior temporal gyrus (STG), ${ }^{14}$ have been also related to specific manifestations of neglect. Severity of neglect seems to depend on the extension of the lesion. $5,15-18$

Different theories on the neural mechanism underlying neglect have been proposed: Various investigators postulated that there is a central deficit in the ability to direct the focus of attention to the left side in right hemisphere patients with neglect. ${ }^{19-21}$ The classical rivalry theory from Mesulam ${ }^{19}$ proposes that the right hemisphere directs attention to both visual hemifields, whereas the left hemisphere directs attention to the right visual field only..$^{19,22}$ Hence, the left hemisphere is unable to compensate for the contralateral damage in the case of a right hemisphere lesion, thereby resulting in visual neglect of the left hemispace. Kinsbourne ${ }^{20}$ proposed a interhemispheric competition theory, developed on the basis that each hemisphere directs attention to the contralateral visual field and is counterbalanced through reciprocal intercallosal inhibition. After a unilateral lesion, the lack of contralateral inhibition provokes the inability of disengaging from the ipsilateral hemispace leading to the neglect syndrome..$^{21,23,24}$ 
Nearly half of all stroke patients are affected by some degree of neglect during acute and subacute phases, ${ }^{25}$ and chronic neglect will be diagnosed in up to $45 \%$ of those patients at 1 year. ${ }^{26}$ Most data on neglect have been gathered from stroke patients and substantially less is known about the incidence of neglect following traumatic brain injury (TBI). A part from few case studies, ${ }^{27,28}$ two retrospective series of patients identified signs of neglect in up to $45 \%$ of TBI patients. ${ }^{29,30}$ The mechanisms of neglect in TBI patients are less well known than in stroke survivors. Only the presence of mass-effect of the lesions has been associated to the presence of neglect. ${ }^{30}$ Interestingly, McKenna and colleagues, ${ }^{29}$ found that the presence of neglect correlated better with worsened motor function than with cognitive impairment. The authors stated that, as in stroke patients, neglect from TBI primary worsens motor function, even though its primary manifestation is in the perceptual sphere.

Visuospatial neglect has been associated with lower performance in sensory and motor abilities ${ }^{31-34}$ in both, stroke patients and TBI survivors. . $^{30,35,36}$

Apart from the impairment itself, neglect complicates treatment, as the syndrome is associated with behavioural and cognitive disabilities that affect the rehabilitation strategies. ${ }^{37,38}$ Since 1970, various approaches to the rehabilitation of visuospatial neglect have been investigated. Initially, rehabilitation used empirical techniques, such as visual or auditory cues in the left hemispace to facilitate gaze shifting towards the left, although those approaches required an effort that many subjects were not able to make. ${ }^{39-41}$ More recently, authors proposed an approach based on bottom up stimulation, which used sensory stimulation maneuvers to modulate the spatial reference system by acting on various sensory afferents such as vestibular and galvanic stimulation ${ }^{42}$ but, even though the effects are evident, they only last for a short period of time. ${ }^{42}$ Prism adaptation is a simple technique which uses the adaptation of sensorimotor inputs to influence high level spatial representation ${ }^{43,44}$ and it has been proved to be effective. ${ }^{45}$ Finally, various pharmacological therapies have been tried with inconsistent results. ${ }^{46}$

More recently, various noninvasive brain stimulation (NIBS) approaches have been explored for the rehabilitation of neglect suffering patients. They are based on the stimulation effects on neuroplasticity and the modulation of neural functions, either facilitating or inhibiting neural networks, including 
synaptogenesis and the generation of changes in the neurons affected, akin to those known as long term potentiation (LTP) and long term depression (LTD) observed in laboratory preparations. ${ }^{47}$

Transcranial direct current stimulation (tDCS) and repetitive transcranial magnetic stimulation (rTMS) are the two most commonly used non-invasive stimulation strategies for visuospatial neglect treatment. tDCS delivers a continuous current (1-2 mA) over the scalp, through anodal and cathodal contacts. ${ }^{48}$ tDCS does not produce action potentials in cortical neurons: it modifies the discharge rate of already active neurons. When the anode is placed over the primary motor cortex, tDCS is thought to increase firing by hyperpolarizing the dendrites and depolarizing the cell body of vertically oriented pyramidal neurons by $<1 \mathrm{mV}$. The opposite effect is observed when the cathode is over the motor cortex. It is postulated that long term effects on corticospinal excitability (after $10 \mathrm{~m}$ or longer stimulation periods) are consequent to the up- or down-regulation of membrane receptors ${ }^{48}$ that lead to LTP- or LTD-like changes in cortical synapses. In addition to the polarity of stimulation, effects are modulated by the duration, intensity and area of stimulation as well as the placement of the electrodes. rTMS is a non-invasive and painless procedure that modulates excitability of cortical motor areas, inducing long-lasting changes in the descending corticospinal tract and transsynaptically at distant sites. High frequency rTMS $(\geq 5 \mathrm{~Hz})$ increases cortical excitability, whereas low frequency rTMS $(\leq 1 \mathrm{~Hz})$ decreases cortical excitability. ${ }^{49}$ Theta burst stimulation (TBS) refers to a rTMS protocol where pulses are applied in bursts of three, delivered at a frequency of $50 \mathrm{~Hz}$, with an inter-burst interval of $200 \mathrm{~ms}(5 \mathrm{~Hz}){ }^{50}$ Intermittent TBS (iTBS) involves the delivery of TBS pulses for 2 s followed by an 8-second rest for a total of about 3 minutes; this is hypothesized to induce LTP-like changes in neural networks, ${ }^{50}$ whereas continuous TBS pulses for 40 seconds (cTBS) generates an effect similar to LTD.

The rationale of the use of rTMS or tDCS in neglect syndrome is the intention to re-establish the altered interhemispheric balance, as postulated by Kinsbourne ${ }^{20,51}$ and corroborated in more recent studies. ${ }^{52}$ The delivery of cathodal current ${ }^{48}$ or low frequency rTMS $^{49,53}$ (or cTBS) on the spared hemisphere is supposed to decrease activity in the targeted cortical region and, 
therefore, diminish the enhanced inhibitory effect over the affected homologous contralateral region. On the other hand, the application of anodal tDCS, ${ }^{48}$ high frequency rTMS or $\mathrm{iTBS}^{49,53}$ on the lesioned side is presumed to have a facilitatory effect on the underlying neurons, so that a functional remapping both online and offline of the perilesional areas is favoured.

Apart from cortical stimulation, peripheral stimulation has been used to treat visuospatial neglect. One such form of peripheral stimulation is neuromuscular vibration (NMV). NMV is a non-invasive stimulation method of the neuromuscular system. Under the definition of vibration therapies, different approaches have been developed: whole body vibration or non-invasive segmental (or focal) muscle vibration. NMV mainly focus on the ability of segmental vibration to modulate neuromuscular system in various neurological conditions. The rationale for using NMV in rehabilitation of neglect is that vibration on neck muscles could modulate sensory inputs from the peripheral sensory organs into body-centered reference allowing for improvement in spatial attention. ${ }^{54}$ Preliminary studies on NMV showed promising results, reorienting the patient's attention when applied on the contralesional side. ${ }^{55}$ Once activated by vibration, muscle spindles generate afferent inputs that can create an illusion of movement, as they are interpreted as a change in muscle length at the corresponding areas of the central nervous system. ${ }^{56}$ When applied to the posterior neck muscles, such NMV-generated illusion can affect the entire egocentric coordinated system, shifting the center of attention towards the side where the NMV is applied. ${ }^{57,58}$ Interestingly, these effects can skip subjective awareness. ${ }^{59-61}$

NMV may induce persistent excitability changes in the implicated central nervous system neuronal circuits $^{62}$ that may be beneficial in various neurological diseases, such as stroke, spinal cord injury multiple sclerosis, Parkinson's' disease and dystonia. ${ }^{57}$ Vibration is well tolerated, effective and easy to use. It could be used to reduce spasticity, to promote motor activity and motor learning, even in gait training, independent from aetiology of the neurological condition. ${ }^{57}$ It is known from previous works that proprioceptive input from the muscle spindles provides information not only for the creation of a subjective map of the body, but also for the orientation of this map in the external world. ${ }^{63}$ Moreover, it is known that segmental muscle vibration induce 
changes in the sensorimotor cortex..$^{64-66}$

The use of NIBS to modulate cortical activity in a target area in patients with neglect was the topic of three recent meta-analysis. ${ }^{67-69}$ However, the authors found no clear positive effect of NIBS for the treatment of neglect following stroke. Some limitations in the scope of these meta-analysis must be pointed out: first, only cerebrovascular disease patients were included; secondly, the authors did not evaluate the type of sham used in the experiments and the blinding of evaluators, and third, the results suffered from bias due to small number of patients and strong heterogeneity of the protocol of NIBS applied with only few randomized controlled studies retrieved. Therefore, we considered that a wider scope of the review may be worth exploring. We decided to add patients with traumatic brain injury (TBI) to those with stroke, as NMV has seen to be effective in patients with TBI. Also, we added NMV to NIBS. Even though NIBS and NMV are two conceptually different treatment strategies for visuospatial neglect, a review of the effects of both may provide more solid ground for an eventual study of the combined effects of the two of them for a more effective therapy of visuospatial neglect, as already pointed out in a very recently published study. ${ }^{70}$

\section{Methods}

We included articles published in English in PubMed, Cochrane Library and MEDLINE databases that explored neglect rehabilitation after acquired brain injuries in adults or pediatric patients up to March 2019. For the literature search, we used the terms "Non-invasive brain stimulation", "non-invasive stimulation", "repetitive transcranial magnetic stimulation (rTMS)", "transcranial direct current stimulation (tDCS)", "vibration", "muscle vibration (NMV)", "neck muscle vibration (NMV)", "segmental vibration", "Neglect", "hemispatial neglect", "visuomotor neglect", "stroke", "traumatic brain injury" and "rehabilitation". We included only studies in which visuospatial neglect was quantified by using cancellation tasks (Albert's task, ${ }^{71}$ Bells test ${ }^{72}$ or other cancellation tests ${ }^{107,109}$ ), the line bisection test (LBT) ${ }^{74}$ or more complex tests such as the behavioural inattention test $(\mathrm{BIT})^{75}$ or the Vienna test. ${ }^{76}$ Articles exploring physiological mechanisms without clinical translation were excluded from the study. 
We included uncontrolled and controlled studies. We defined a study as controlled if the study designed involved a control condition, either in the form of healthy subjects or placebo groups. Controlled studies were divided between randomized and non-randomized, according to whether or not a randomization process was described in the manuscript. We further separated reports of single cases or a small group of patients (less than 3) from studies including a larger group of patients and, lastly, blinded from unblinded studies, including them in the blinding group when at least the patients were blinded to treatment (Figure $1)$.

We examined demographic and clinical features of the patients (age, time since injury onset, number of subjects), stimulation characteristics (study design, NIBS or NMV protocol, site of stimulation, neglect assessment, outcome and follow up) and concomitant treatments including conventional rehabilitation (Table 1 and 2).

\section{Results}

Thirty-four studies were finally included that matched our search criteria. 10,55,84-93,58,94-103,77,104-107,78-83 Figure 1 shows a schematic distribution of the studies according to the groups defined above. Tables 1 and 2 summarize the main points evaluated in each study. The number of participants in the studies varied widely from 1 to 32 patients. Patients' age ranged from 20 to 84 years. In 14 studies, $55,79,102,104,105,107,82,83,85,91,97-99,101$ the authors reported concomitant treatments such as occupational therapy, prism adaptation or other rehabilitation approaches. Most studies were performed during the chronic phase of the acquired brain injury (ABI). ${ }^{10,58,95,97,99,102,106,107,78,79,81,82,84,87,90,93}$ All studies were conducted in adult stroke patients, except for one in a patient with $\mathrm{TBI}^{84}$ and another in a patient with brain tumor. ${ }^{58}$. No side effects were reported in any of the studies.

Clinical impairment, measured with acute stroke scales, was usually severe, ${ }^{58,91}$ although scarce clinical data other than neglect were usually supplied. The affected hemisphere was the right in all the studies retrieved. The severity of the neglect was clearly reported in only 7 studies $(20.6 \%)^{55,58,81,89,91,101,106}$ and was classified as severe in all of them (table 1$)$. 
Transcranial Magnetic stimulation (TMS):

The effects of rTMS on neglect were reported in 16 articles. ${ }^{77,78,88,102-}$ $105,108,79-84,86,87$ In all of them, rTMS was applied over the parietal cortex of the unaffected hemisphere. In the majority of the studies, the authors applied inhibitory stimulation, such as LFrTMS (7 studies; $43.5 \%)^{77-80,82,85,87,103}$ or cTBS (7 studies; 43.5\%). $81,83,84,86,102,104,105$ HFrTMS was used as a stand-alone treatment method in only one open label study $(6.5 \%)^{93}$ and iTBS was applied over the dorsolateral prefrontal cortex (DLPFC) in another study. ${ }^{77}$

In most studies $55,79,100-102,104,105,107,108,82,83,86,91,93,96-98$, patients continued other forms of therapy, while rTMS was added-on. In these instances, significantly more improvement is reported with the addition of rTMS than with conventional therapy alone, although no information is given on whether the effect was maintained or not. The number of therapy sessions used ranged between 1 and 20 in a two weeks period. The usual session duration was 30 minutes (table 2), except for TBS sessions, which duration was less than 1 minute. The number of pulses per session varied between 600 and 1200 (table 2). A true sham coil was used in only 4 studies $(25 \%), 81,83,87,104$ while other sham strategies included a reversed coil102 (scalp facing the "inactive" side of the coil) or holding the coil perpendicular to the scalp. ${ }^{77,85,86,103,105}$.

Some degree of improvement in the tests used to assess neglect was reported in all studies. In some of them, neuroimaging methods were also used to examine a possible surrogate of the clinical changes: Shindo et al. ${ }^{79}$ failed to show a significant change in brain activity by single photon emission tomography performed just after the last session. Bonni et al ${ }^{84}$ showed a bilateral increase of functional connectivity in the frontoparietal network in 1 patient suffering neglect after TBI who underwent 7 sessions of cTBS over the left parietal cortex (P3). Cao et al. ${ }^{88}$ found a large-scale reduction in the resting state $\mathrm{FMRI}$ connectivity extent in the right attention network in patients receiving iTBS over the left DLPFC. In a prospective, double-blind, sham-controlled trial, Kim et al. ${ }^{85}$ compared LFrTMS over the unaffected parietal cortex versus HFrTMS over the affected parietal cortex and found no differences between the two forms of real stimulation, which were significantly more effective than sham stimulation in several neglect-assessment tests, although patients reported no clear improvement in daily life activity. 
In several randomized sham-controlled LFrTMS studies $80,87,102,103,108$, the authors reported improvement of neglect for up to 1 week after treatment. In those using cTBS $83,86,102,104,105$ the authors found improvement that lasted a variable time after treatment, ranging from 2 weeks after 2 daily sessions for 10 days ${ }^{105}$, to 4 weeks after 2 daily sessions for 2 weeks ${ }^{86}$. A modified cTBS protocol was investigated by Nyffeler and colleagues. ${ }^{81}$ The protocol consisted of 801 pulses delivered in 267 bursts of 3 pulses at $30 \mathrm{~Hz}$, repeated every 100 $\mathrm{ms}$. The authors reported a benefit that lasted up to 32 hours after a single session of 4 TBS trains. Using the same protocol but doubling the number of TBS trains (4 each day in two consecutive days), Cazzoli et al. ${ }^{83}$ found a strong improvement in test performance that persisted 3 weeks after the treatment.

HFrTMS over the unaffected hemisphere was used as a stand-alone method for treatment by Oliveri et al. ${ }^{77}$ in an uncontrolled, open-label, study. These authors intended to produce a transient virtual lesion to diminish the inhibitory effect over the homologous area in the affected hemisphere. The beneficial effects were short-lasting. More recently, Yang et al ${ }^{102}$ compared three different rTMS modalities, delivered on the left hemisphere, LFrTMS, HFrTMS and cTBS, against sham, in 38 stroke patients. All treatments lasted for 2 weeks while patients were engaged in an intense rehabilitation therapy program. The authors found that CTBS, applied according to the protocol described above for Nyfeller et al., ${ }^{81}$ produced the stronger effect, followed by LFrTMS and, then, HFrTMS, which all were significantly different from sham. The improvement was still present at 1 month follow-up.

\section{tDCS}

The effects of tDCS on neglect were reported in 10 articles. ${ }^{10,89-}$ $94,101,106,107$ In 3 of them, the authors explored the effects of anodal current over the affected parietal cortex with the cathode over the contralateral supraorbital region. ${ }^{89,93,94,101,106}$ In the remaining studies, the anode was placed over the affected posterior parietal cortex and the cathode over the contralateral, unaffected, area. ${ }^{10,90,92,107}$ The number of therapy sessions ranged from 1 to 15 and the average duration of each session was 20 minutes. The intensity used was between 1 and $2 \mathrm{~mA}$. All studies included a control group either as a 
separate sham stimulation group or in a cross-over design. All but one study, ${ }^{106}$ showed some degree of improvement in hemineglect syndrome.

Sparing et al. ${ }^{10}$ used a cross-over study design to investigate the effects of anodal vs cathodal stimulation. Ten patients with right hemispheric stroke received anodal or cathodal stimulation over the unaffected hemisphere, anodal stimulation over the affected hemisphere or placebo stimulation. All the stimulations were performed on different days in a randomized fashion. The authors found that both the cathodal tDCS applied over the unaffected hemisphere and anodal tDCS applied over the affected hemisphere ameliorated hemi-spatial neglect. Similar methods were used by Ladavas et al., ${ }^{107}$ who demonstrated that only anodal stimulation over the right parietal cortex let to a significantly more improvement in the prism adaptation task than sham, while no amelioration beyond sham was seen in the cathodal stimulation over the left parietal cortex.

Sunwoo et al. ${ }^{90}$ compared the effects of dual stimulation (anode over the affected parietal cortex and cathode over the unaffected parietal cortex) with single anodal stimulation (anode over the affected parietal cortex and cathode over the contralateral supraorbital area) and concluded that both stimulation modalities led to improvement, but this was significantly larger with the dual stimulation method than with the single anodal stimulation method. Biparietal tDCS was also found to potentiate the beneficial effects of rehabilitation in a double blind, sham-controlled study of a single case, ${ }^{91}$ as well as of an optokinetic task in a group of 32 patients. ${ }^{101}$ In both studies, the effect was maintained for some time after treatment: up to 1 month in the single patient and up to 6 days as a mean in the group study. A similar benefit of combined therapies was reported by Bang et al. ${ }^{93}$ using just anodal stimulation of the left parietal cortex (with cathode placed over the contralateral supraorbital area). The study was carried out in a group of patients submitted to feedback training, who exhibited a significant improvement in all measuring tests when real tDCS was added to the treatment.

Smit et al. ${ }^{106}$ carried out a double-blind, placebo-controlled study of the effects of a biparietal stimulation montage (anode on P4 and cathode on P3) in 5 patients with severe chronic hemispatial neglect, after exclusion of a large number of patients because of contraindications for stimulation, presence of 
other medical conditions or only mild neglect. In their study, these authors did not find differences between real tDCS or placebo, applied for 20 minutes with an intensity of $2 \mathrm{~mA}$ during 5 consecutive days.

NMV

The effects of NMV on neglect were reported in 8 articles. ${ }^{58,61,84-89}$ They were all carried out in chronic stroke patients, with a study population varying from a single subject to 20 patients. The vibration utilized ranged from 80 to $600 \mathrm{~Hz}$ and the most common used site of treatment delivery was the posterior neck muscles contralateral to the affected hemisphere. Only 3 studies (37.5\%) repeated the NMV sessions more than once. ${ }^{55,98,100}$ In most studies, NMV was applied in addition to various forms of neglect therapy. In 5 studies, ${ }^{95-}$ 97,99,100 such therapy was used as a control condition and, in all of them, there was significantly more improvement with NMV. Duration of the improvement was up to 1.4 years in 6 patients studied by Johanssen et al. ${ }^{98}$

Karnath et al. ${ }^{95}$ examined the effects of neck muscle NMV on neglect symptoms in three patients with right hemisphere lesions. The authors used different conditions of trunk rotation while the patients were asked to identify visual stimuli in both hemi-visual fields. They found that NMV applied to the left side of the neck improved the detection of the stimuli presented, hypothesizing a shift of the body centre induced by the applied vibration. To further investigate their results, the same authors compared contralateral neck vibration with electrical stimulation and hand vibration ${ }^{96}$. Only the neck vibration significantly improved the performance in cancellation and copying tasks, with no or little effect of the other modalities. The authors hypothesized that the la fibres are directly involved in representation of the egocentric space.

\section{Discussion}

Our systematic review explores the use of non-invasive stimulation technics which includes brain stimulation (NIBS) and muscle vibration stimulations (NMV) to improve neglect syndrome following acquired brain injury. Almost all non-invasive stimulation treatments (rTMS, tDCS or NMV) included in 
this review reported a significant improvement of neglect in observational as well as in controlled studies. The single exception being the lack of tDCS effect reported by Smit et al. ${ }^{106}$ in a group of 5 patients with severe chronic neglect after a right hemisphere stroke. On the other side, no-one reported improvement in control or sham group. Randomized studies including placebo group are important to validate the effectiveness of the neuromodulation therapies. Our study has shown more methodological variability in the approach to the design of the control condition in rTMS and NMV studies than in TDCS studies, but results were consistently similar in all therapies.

In overall, results were promising for improvement of neglect regardless of (i) whether the stimulation is applied to the brain or to the muscle, (ii) what stimulation technique is used; (iii) whether the study is controlled, blinded or randomized. Remarkably, however, only one study ${ }^{83}$ explored the effects of treatment in daily life activities, while only two studies reported improvement in functional scales such as Catherine Berego Scale. ${ }^{85,92}$

In most instances, brain stimulation was applied in order to inhibit activity in the unaffected hemisphere: LFrTMS or cathodal tDCS over the unaffected parietal cortex (P3-P5, according to EEG nomenclature). Non-invasive brain stimulation for stroke is based on the interhemispheric competition model facilitating ipsilesional cortical excitability and suppressing contralesional cortical excitability for balancing cortical excitability and interhemispheric inhibition levels between hemispheres. ${ }^{109-111}$ In these studies, rTMS and tDCS were predominantly used to decrease the hyperactivity of the unaffected hemisphere, hence diminishing its excess inhibitory effect over the damaged hemisphere. ${ }^{49}$ In fact, unilateral neglect is thought to be secondary to an imbalance in interhemispheric rivalry. ${ }^{112}$ The decrease in interhemispheric inhibition from the affected to the unaffected hemisphere may lead to an exaggerated activity of the healthy hemisphere, which in turn force the deviation of the attention of the subject from the affected hemibody towards the contralateral one. ${ }^{25}$ Brain stimulation therapies are intended to restore the disrupted interhemispheric balance by inhibiting the unaffected hemisphere, ${ }^{49}$ activating the affected hemisphere or both. NMV is intended to increase the saliency of sensory inputs to the lesioned hemisphere ${ }^{57}$ and potentiate in this way the patient's awareness of the contralateral hemi-space. 
The following points determine the potential interest of the present review for future studies:

A) There was a substantial variability of the size of study population, varying between single case studies and a study group of up to 32 patients.

B) There is a scarcity of studies on efficacy and clinical relevance of the results for daily life activity, data being limited to experimental tests.

C) The associated neurological deficits and comorbidities are not usually considered.

D) The results obtained with rTMS and tDCS are consistent with the pathophysiological concepts derived from the theory of interhemispheric competition and, therefore, favor these pathophysiological mechanisms as those underlying neglect.

E) There is need for refinement of stimulation methods for rTMS (type, intensity, duration and frequency of the sessions), tDCS (number of sessions, duration, exact location) and NMV (frequency, stimulation site and side, number of sessions) to define the optimal stimulation protocols.

F) The safety and secondary effects of long-term effects of repeated sessions for rTMS, tDCS and NMV should be considered in relation to the benefit obtained. This mainly refers to long term follow-up, which has not been longer than 6 weeks in most of the studies reviewed.

G) Optimal post-stroke delay (acute vs chronic state) has not been defined yet, in terms of effectiveness or harmlessness.

$\mathrm{H})$ The selection of patients that could benefit more from stimulation treatments must consider location and size of the lesion, clinical characteristics, severity of hemineglect.

I) Combined care management within a neuromodulation approach should be explored.

Our results complement those published in previous metanalysis on the efficacy of NIBS for treatment of unilateral neglect. ${ }^{67-69}$ Although the authors found mild evidence of the efficacy of rTMS for the treatment of neglect, no 
significant positive effect was found regarding tDCS. Furthermore, recently 1 systematic review on TBS and neglect ${ }^{113}$ found a positive effect on the symptoms although a strong heterogeneity of protocols and assessment was found. As stated, only cerebrovascular disease patients were included, the results were biased by the small number of patients and strong heterogeneity of the protocol of NIBS.

Consequently, we widened the scope of the previous reviews adding, patients suffering brain tumor and traumatic brain injury, and, more importantly we included studies exploring the use of NMV for neglect treatment.

Recently Perasso et al. ${ }^{70}$ investigated the possibility to consolidate the cortical aftereffect of NMV by combining tendon vibration with a concomitant highfrequency $5-\mathrm{Hz}$ rTMS protocol. The group found that this protocol induced a pattern of unbalanced M1 excitability between vibrated muscle and its antagonist with increased excitability of the agonist and decreased excitability of the antagonist muscle cortical areas, which persisted up to $30 \mathrm{~min}$, demonstrating a synergic effect. Those neuromodulatory strategies have a different mechanism of action, although a synergic effect might hypothetically exist, given the mostly top down use of NIBS and the bottom up effect of NMV. Thus, the combination of different therapies, based on complementary and synergic effects might be a new and important field of investigation.

In conclusion, non-invasive stimulation, either of the brain (rTMS or tDCS) or the muscle (NMV) is a promising addition to the treatment of neglect. In addition, non-invasive stimulation tools combined with specific training methods could induce sustainable and functional improvement. There is a need for further investigations with randomized sham controlled trials that include large samples and evaluate the long term efficacy of repeated sessions of non-invasive stimulation procedures. The combination of rTMS or tDCS with NMV is of special interest, as it has demonstrated its feasibility in a recent study. ${ }^{76}$ Future studies should include the evaluation of effects on activities of daily living and the potential transfer to daily life situations.

\section{Clinical Message}


- Non-invasive brain modulation and neuromuscular vibration are effective tools to improve neglect after stroke although the heterogeneity of studies is high.

- Combination of different treatments may improve the outcome

- Further randomized controlled large-sample trials are needed

Conflicting interests:

The Author(s) declare(s) that there is no conflict of interest Funding:

This research received no specific grant from any funding agency in the public, commercial, or not-for-profit sectors.

Contributorship:

GL, NM and HK designed the study and reviewed de literature; JVS,JR AD and JC revised the manuscript and corrected the proof.

All authors reviewed and edited the manuscript and approved the final version of the manuscript.

Acknowledgements: None 


\section{References}

1. Stone SP, Halligan PW, Marshall JC, et al. Unilateral neglect: a common but heterogeneous syndrome. Neurology 1998; 50: 1902-5.

2. Laplane D, Degos JD. Motor neglect. J Neurol Neurosurg Psychiatry 1983; 46: 152-158.

3. Vallar G, Bottini G, Paulesu E. Neglect syndromes: the role of the parietal cortex. Adv Neurol 2003; 93: 293-319.

4. Bisiach E, Perani D, Vallar G, et al. Unilateral neglect: personal and extrapersonal. Neuropsychologia 1986; 24: 759-67.

5. Vallar G, Perani D. THE ANATOMY OF UNILATERAL NEGLECT AFTER RIGHT- HEMISPHERE STROKE LESIONS . A CLINICAL / CT-SCAN CORRELATION STUDY IN MAN. Neuropsycologia 1986; 24: 609-622.

6. Robertson IH, North N. Active and passive activation of left limbs: influence on visual and sensory neglect. Neuropsychologia 1993; 31: 293-300.

7. Driver J, Mattingley JB. Parietal neglect and visual awareness. Nat Neurosci 1998; 1: 17-22.

8. Mesulam MM. Spatial attention and neglect: parietal, frontal and cingulate contributions to the mental representation and attentional targeting of salient extrapersonal events. Philos Trans R Soc Lond B Biol Sci 1999; 354: 1325-46.

9. Kerkhoff G. Spatial hemineglect in humans. Prog Neurobiol 2001; 63: 127.

10. Sparing R, Thimm M, Hesse MD, et al. Bidirectional alterations of interhemispheric parietal balance by non-invasive cortical stimulation. Brain 2009; 132: 3011-3020.

11. Bowen A, McKenna K, Tallis RC. Reasons for variability in the reported rate of occurrence of unilateral spatial neglect after stroke. Stroke 1999; 30: 1196-202.

12. Husain M, Kennard C. Distractor-dependent frontal neglect. Neuropsychologia 1997; 35: 829-41.

13. Vallar G. Extrapersonal Visual Unilateral Spatial Neglect and Its Neuroanatomy. Neuroimage 2001; 14: S52-S58.

14. Karnath $\mathrm{H}-\mathrm{O}$. New insights into the functions of the superior temporal 
cortex. Nat Rev Neurosci 2001; 2: 568-576.

15. Mort DJ, Malhotra P, Mannan SK, et al. The anatomy of visual neglect. Brain 2003; 126: 1986-1997.

16. Karnath $\mathrm{H}-\mathrm{O}$, Fruhmann Berger M, Küker W, et al. The Anatomy of Spatial Neglect based on Voxelwise Statistical Analysis: A Study of 140 Patients. Cereb Cortex 2004; 14: 1164-1172.

17. Thimm M, Fink GR, Sturm W. Neural correlates of recovery from acute hemispatial neglect. Restor Neurol Neurosci 2008; 26: 481-92.

18. Corbetta $M$, Kincade MJ, Lewis $C$, et al. Neural basis and recovery of spatial attention deficits in spatial neglect. Nat Neurosci 2005; 8: 16031610.

19. Mesulam M-M. A cortical network for directed attention and unilateral neglect. Ann Neurol 1981; 10: 309-325.

20. Kinsbourne M. Hemi-neglect and hemisphere rivalry. Adv Neurol 1977; 18: 41-9.

21. Kinsbourne M. Integrated cortical field model of consciousness. Ciba Found Symp 1993; 174: 43-50; discussion 51-60.

22. Heilman KM, Van Den Abell T. Right hemisphere dominance for attention: the mechanism underlying hemispheric asymmetries of inattention (neglect). Neurology 1980; 30: 327-30.

23. Cohen JD, Romero RD, Servan-Schreiber D, et al. Mechanisms of Spatial Attention: The Relation of Macrostructure to Microstructure in Parietal Neglect. J Cogn Neurosci 1994; 6: 377-387.

24. Smania N, Martini MC, Gambina G, et al. The spatial distribution of visual attention in hemineglect and extinction patients. Brain 1998; 121: 17591770.

25. Husain M. Hemispatial neglect. Handb Clin Neurol Neuropsychol Behav Neurol 2008; 88: 359-72.

26. Ringman JM, Saver JL, Woolson RF, et al. Frequency, risk factors, anatomy, and course of unilateral neglect in an acute stroke cohort. Neurology 2004; 63: 468-474.

27. Halterman $\mathrm{Cl}$, Langan J, Drew A, et al. Tracking the recovery of visuospatial attention deficits in mild traumatic brain injury. Brain 2006; 129: 747-753. 
28. Van Donkelaar P, Langan J, Rodriguez E, et al. Attentional deficits in concussion. Brain Inj 2005; 19: 1031-1039.

29. McKenna K, Cooke DM, Fleming J, et al. The incidence of visual perceptual impairment in patients with severe traumatic brain injury. Brain Inj 2006; 20: 507-518.

30. Chen P, Ward I, Khan U, et al. Spatial Neglect Hinders Success of Inpatient Rehabilitation in Individuals With Traumatic Brain Injury. Neurorehabil Neural Repair 2016; 30: 451-460.

31. Nijboer T, van de Port I, Schepers V, et al. Predicting Functional Outcome after Stroke: The Influence of Neglect on Basic Activities in Daily Living. Front Hum Neurosci; 7. Epub ahead of print 2013. DOI: 10.3389/fnhum.2013.00182.

32. Kimura Y, Yamada M, Ishiyama D, et al. Impact of unilateral spatial neglect with or without other cognitive impairments on independent gait recovery in stroke survivors. J Rehabil Med 2019; 51: 26-31.

33. Sobrinho KRF, Santini ACM, Marques CLS, et al. Impact of unilateral spatial neglect on chronic patient's post-stroke quality of life. Somatosens Mot Res 2018; 35: 199-203.

34. Fasotti L, van Kessel M. Novel Insights in the Rehabilitation of Neglect. Front Hum Neurosci 2013; 7: 1-8.

35. Katz N, Hartman-Maeir A, Ring H, et al. Functional disability and rehabilitation outcome in right hemisphere damaged patients with and without unilateral spatial neglect. Arch Phys Med Rehabil 1999; 80: 379_ 84.

36. Denes G, Semenza C, Stoppa E, et al. Unilateral spatial neglect and recovery from hemiplegia: a follow-up study. Brain 1982; 105 (Pt 3): 54352.

37. Arene NU, Hillis AE. Rehabilitation of unilateral spatial neglect and neuroimaging. Eura Medicophys 2007; 43: 255-69.

38. Di Monaco M, Schintu S, Dotta M, et al. Severity of unilateral spatial neglect is an independent predictor of functional outcome after acute inpatient rehabilitation in individuals with right hemispheric stroke. Arch Phys Med Rehabil 2011; 92: 1250-6.

39. Diller L, Weinberg J. Hemi-inattention in rehabilitation: the evolution of a 
rational remediation program. Adv Neurol 1977; 18: 63-82.

40. Webster JS, Jones $\mathrm{S}$, Blanton $\mathrm{P}$, et al. Visual scanning training with stroke patients. Behav Ther 1984; 15: 129-143.

41. Smania N, Bazoli F, Piva D, et al. Visuomotor imagery and rehabilitation of neglect. Arch Phys Med Rehabil 1997; 78: 430-436.

42. Rossetti $Y$, Rode $G$. Reducing spatial neglect by visual and other sensory manipulations: non-cognitive (physiological) routes to the rehabilitation of a cognitive disorder. In: Karnath $\mathrm{H}-\mathrm{O}$, Milner D, Vallar G (eds) The Cognitive and Neural Bases of Spatial Neglect. Oxford University Press, 2002.

43. Rossetti; LPRFT. Prism adaptation in the rehabilitation of patients with visuo-spatial cognitive disorders. Curr Opin Neurol 2006; 19: 534-542.

44. Luauté J, Jacquin-Courtois S, O'Shea J, et al. Left-Deviating Prism Adaptation in Left Neglect Patient: Reflexions on a Negative Result. Neural Plast 2012; 2012: 1-10.

45. Shiraishi $H$, Yamakawa $Y$, Itou $A$, et al. Long-term effects of prism adaptation on chronic neglect after stroke. NeuroRehabilitation 2008; 23 : 137-51.

46. Singh-Curry V, Husain M. Rehabilitation in practice: Hemispatial neglect: approaches to rehabilitation. Clin Rehabil 2010; 24: 675-684.

47. Lefaucheur JP, André-Obadia N, Antal A, et al. Evidence-based guidelines on the therapeutic use of repetitive transcranial magnetic stimulation (rTMS). Clinical Neurophysiology 2014; 125: 2150-2206.

48. Lang N, Nitsche MA, Paulus W, et al. Effects of transcranial direct current stimulation over the human motor cortex on corticospinal and transcallosal excitability. Exp Brain Res 2004; 156: 439-443.

49. Rossi S, Hallett M, Rossini PM, et al. Safety, ethical considerations, and application guidelines for the use of transcranial magnetic stimulation in clinical practice and research q. Clin Neurophysiol 2009; 120: 2008-2039.

50. Rossini PM, Burke D, Chen R, et al. Clinical Neurophysiology Noninvasive electrical and magnetic stimulation of the brain, spinal cord, roots and peripheral nerves: Basic principles and procedures for routine clinical and research application. An updated report from an. Clin Neurophysiol 2015; 126: 1071-1107. 
51. Kinsbourne M, Bruce R. Shift in visual laterality within blocks of trials. Acta Psychol (Amst) 1987; 66: 139-55.

52. Pascual-Leone A, Amedi A, Fregni F, et al. THE PLASTIC HUMAN BRAIN CORTEX. Annu Rev Neurosci 2005; 28: 377-401.

53. Rossini PM, Burke D, Chen R, et al. Non-invasive electrical and magnetic stimulation of the brain, spinal cord, roots and peripheral nerves: Basic principles and procedures for routine clinical and research application. An updated report from an I.F.C.N. Committee. Clin Neurophysiol 2015; 126 : 1071-1107.

54. Rosenkranz K, Rothwell JC. Differential effect of muscle vibration on intracortical inhibitory circuits in humans. J Physiol 2003; 551: 649-60.

55. Pitteri M, Arcara G, Passarini L, et al. Is Two Better than One? Limb Activation Treatment Combined with Contralesional Arm Vibration to Ameliorate Signs of Left Neglect. Front Hum Neurosci 2013; 7: 1-10.

56. Goodwin GM, McCloskey DI, Matthews PB. Proprioceptive illusions induced by muscle vibration: contribution by muscle spindles to perception? Science 1972; 175: 1382-4.

57. Leplaideur S, Leblong E, Jamal K, et al. Short-term effect of neck muscle vibration on postural disturbances in stroke patients. Exp Brain Res 2016; 234: 2643-2651.

58. Ceyte $\mathrm{H}$, Beis JM, Simon M, et al. Lasting improvements in left spatial neglect following a protocol combining neck-muscle vibration and voluntary arm movements: a case-study. Disabil Rehabil 2019; 41: 14751483.

59. Ceyte $\mathrm{H}$, Cian $\mathrm{C}$, Nougier V, et al. Effects of neck muscles vibration on the perception of the head and trunk midline position. Exp Brain Res 2006; 170: 136-140.

60. Guinet M, Michel C. Prism adaptation and neck muscle vibration in healthy individuals: Are two methods better than one? Neuroscience 2013; 254: 443-451.

61. Kerkhoff $\mathrm{G}$. Modulation and rehabilitation of spatial neglect by sensory stimulation. In: Progress in brain research, pp. 257-271.

62. Forner-Cordero A, Steyvers M, Levin O, et al. Changes in corticomotor excitability following prolonged muscle tendon vibration. Behav Brain Res 
2008; 190: 41-49.

63. Matthews PB. Proprioceptors and their contribution to somatosensory mapping: complex messages require complex processing. Can J Physiol Pharmacol 1988; 66: 430-8.

64. Lapole T, Temesi J, Gimenez P, et al. Achilles tendon vibration-induced changes in plantar flexor corticospinal excitability. Exp Brain Res 2015; 233: 441-448.

65. Seo NJ, Lakshminarayanan K, Lauer AW, et al. Use of imperceptible wrist vibration to modulate sensorimotor cortical activity. Exp brain Res 2019; 237: 805-816.

66. Lopez S, Bini F, Del Percio C, et al. Electroencephalographic sensorimotor rhythms are modulated in the acute phase following focal vibration in healthy subjects. Neuroscience 2017; 352: 236-248.

67. Fan J, Li Y, Yang Y, et al. Efficacy of Noninvasive Brain Stimulation on Unilateral Neglect After Stroke. Am J Phys Med Rehabil 2018; 97: 261269.

68. Salazar APS, Vaz PG, Marchese RR, et al. Noninvasive Brain Stimulation Improves Hemispatial Neglect After Stroke: A Systematic Review and Meta-Analysis. Arch Phys Med Rehabil 2018; 99: 355-366.e1.

69. Kashiwagi FT, El Dib R, Gomaa H, et al. Noninvasive Brain Stimulations for Unilateral Spatial Neglect after Stroke: A Systematic Review and Meta-Analysis of Randomized and Nonrandomized Controlled Trials. Neural Plast 2018; 2018: 1-25.

70. Perasso L, Avanzino L, Lagravinese G, et al. Boosting and consolidating the proprioceptive cortical aftereffect by combining tendon vibration and repetitive TMS over primary motor cortex. Neurol Sci 2019; 40: 147-154.

71. Albert M. A simple test for visual neglect. Neurology 1973; 23: 658-64.

72. Gauthier $L$, Dehaut $F$, Joanette $Y$. The bells test: a quantitative and qualitative test for visual neglect. Int J Neuropsycology 1989; 11: 49-54.

73. Mesulam, MM Weintraub S. Mental state assessment of young and elderly adults in behavioural neurology. In: Principles of behavioural neurology. 1985, pp. 71-123.

74. Schenkenberg T, Bradford DC, Ajax ET. Line bisection and unilateral visual neglect in patients with neurologic impairment. Neurology 1980; 30: 
509-17.

75. Wilson B, Cockburn J, Halligan P. Development of a behavioral inattention test of visuospatial neglect. Arch Phys Med Rehabil 1987; 68: 98-102.

76. Schufried G. Vienna Test System: Cognitrone.

77. Oliveri M, Bisiach E, Brighina F, et al. rTMS of the unaffected hemisphere transiently reduces contralesional visuospatial hemineglect. Neurology 2001; 57: 1338-1340.

78. Brighina $F$, Bisiach $\mathrm{E}$, Oliveri $\mathrm{M}$, et al. $1 \mathrm{~Hz}$ repetitive transcranial magnetic stimulation of the unaffected hemisphere ameliorates contralesional visuospatial neglect in humans. Neurosci Lett 2003; 336: 131-133.

79. Shindo K, Sugiyama K, Huabao L, et al. Long-term effect of lowfrequency repetitive transcranial magnetic stimulation over the unaffected posterior parietal cortex in patients with unilateral spatial neglect. $J$ Rehabil Med 2006; 38: 65-67.

80. Song $W, D u B, X u Q$, et al. Low-frequency transcranial magnetic stimulation for visual spatial neglect: A pilot study. J Rehabil Med 2009; 41: 162-165.

81. Nyffeler T, Cazzoli D, Hess CW, et al. One session of repeated parietal theta burst stimulation trains induces long-lasting improvement of visual neglect. Stroke 2009; 40: 2791-2796.

82. Lim J, Kang E, Paik N. Repetitive transcranial magnetic stimulation to hemispatial neglect in patients after stroke: An open-label pilot study. $J$ Rehabil Med 2010; 42: 447-452.

83. Cazzoli D, Müri RM, Schumacher R, et al. Theta burst stimulation reduces disability during the activities of daily living in spatial neglect. Brain 2012; 135: 3426-3439.

84. Bonnì $\mathrm{S}$, Mastropasqua $\mathrm{C}$, Bozzali $\mathrm{M}$, et al. Theta burst stimulation improves visuo-spatial attention in a patient with traumatic brain injury. Neurol Sci 2013; 34: 2053-2056.

85. Kim BR, Chun MH, Kim DY, et al. Effect of high- and low-frequency repetitive transcranial magnetic stimulation on visuospatial neglect in patients with acute stroke: A double-blind, sham-controlled trial. Arch 
Phys Med Rehabil 2013; 94: 803-807.

86. Fu W, Song W, Zhang $\mathrm{Y}$, et al. Long-term effects of continuous thetaburst stimulation in visuospatial neglect. J Int Med Res 2015; 43: 196203.

87. Cha HG, Kim MK. Effects of repetitive transcranial magnetic stimulation on arm function and decreasing unilateral spatial neglect in subacute stroke: A randomized controlled trial. Clin Rehabil 2016; 30: 649-656.

88. Cao L, Fu W, Zhang $\mathrm{Y}$, et al. Intermittent $\theta$ burst stimulation modulates resting-state functional connectivity in the attention network and promotes behavioral recovery in patients with visual spatial neglect. Neuroreport 2016; 27: 1261-1265.

89. Ko MH, Han SH, Park SH, et al. Improvement of visual scanning after DC brain polarization of parietal cortex in stroke patients with spatial neglect. Neurosci Lett 2008; 448: 171-174.

90. Sunwoo H, Kim YH, Chang WH, et al. Effects of dual transcranial direct current stimulation on post-stroke unilateral visuospatial neglect. Neurosci Lett 2013; 554: 94-98.

91. Brem A-K, Unterburger E, Speight I, et al. Treatment of visuospatial neglect with biparietal tDCS and cognitive training: a single-case study. Front Syst Neurosci 2014; 8: 1-9.

92. Yi YG, Chun MH, Do KH, et al. The effect of transcranial direct current stimulation on neglect syndrome in stroke patients. Ann Rehabil Med 2016; 40: 223-229.

93. Bang D-H, Bong S-Y. Effect of combination of transcranial direct current stimulation and feedback training on visuospatial neglect in patients with subacute stroke: a pilot randomized controlled trial. J Phys Ther Sci 2015; 27: 2759-2761.

94. Bornheim S, Maquet $\mathrm{P}$, Croisier JL, et al. Motor cortex Transcranial Direct Current Stimulation (tDCS) improves acute stroke visuo-spatial neglect: A series of four case reports. Brain Stimulation, 2018, pp. 459-461.

95. Karnath HO, Christ K, Hartje W. Decrease of unilateral neglect by neck muscle vibration and spatial orientation of the trunk midline. Brain 1993; 116: 383-396.

96. Karnath $\mathrm{HO}$. Transcutaneous electrical stimulation and vibration of neck 
muscles in neglect. Exp Brain Res 1995; 105: 321-324.

97. Schindler I, Kerkhoff G, Karnath $\mathrm{HO}$, et al. Neck muscle vibration induces long lasting recovery in spatial neglect. J Neurol Neurosurg Psych 2002; 73: 412-419.

98. Johannsen L, Ackermann H, Karnath HO. Lasting amelioration of spatial neglect by treatment with neck muscle vibration even without concurrent training. J Rehabil Med 2003; 35: 249-253.

99. Saevarsson S, Kristjansson A, Halsband U. Strength in numbers: Combining neck vibration and prism adaptation produces additive therapeutic effects in unilateral neglect. Neuropsychol Rehabil 2010; 20: 704-724.

100. Kamada K, Shimodozono M, Hamada H, et al. Effects of 5 minutes of neck-muscle vibration immediately before occupational therapy on unilateral spatial neglect. Disabil Rehabil 2011; 33: 2322-2328.

101. Turgut N, Miranda M, Kastrup A, et al. tDCS combined with optokinetic drift reduces egocentric neglect in severely impaired post-acute patients. Neuropsychol Rehabil 2018; 28: 515-526.

102. Yang W, Liu TT, Song $X$ Bin, et al. Comparison of different stimulation parameters of repetitive transcranial magnetic stimulation for unilateral spatial neglect in stroke patients. J Neurol Sci 2015; 359: 219-225.

103. Agosta S, Herpich F, Miceli G, et al. Contralesional rTMS relieves visual extinction in chronic stroke. Neuropsychologia 2014; 62: 269-276.

104. Hopfner S, Cazzoli D, Müri RM, et al. Enhancing treatment effects by combining continuous theta burst stimulation with smooth pursuit training. Neuropsychologia 2015; 74: 145-151.

105. Koch G, Bonnì S, Giacobbe V, et al. Theta-burst stimulation of the left hemisphere accelerates recovery of hemispatial neglect. Neurology 2012; 78: 24-30.

106. Smit M, Schutter DJLG, Nijboer TCW, et al. Transcranial direct current stimulation to the parietal cortex in hemispatial neglect: A feasibility study. Neuropsychologia 2015; 74: 152-161.

107. Làdavas $E$, Giulietti $S$, Avenanti $A$, et al. A-tDCS on the ipsilesional parietal cortex boosts the effects of prism adaptation treatment in neglect. Restor Neurol Neurosci 2015; 33: 647-662. 
108. Kim YK, Jung JH, Shin SH. A comparison of the effects of repetitive transcranial magnetic stimulation (rTMS) by number of stimulation sessions on hemispatial neglect in chronic stroke patients. Exp Brain Res 2014; 233: 283-289.

109. Murase N, Duque J, Mazzocchio R, et al. Influence of interhemispheric interactions on motor function in chronic stroke. Ann Neurol 2004; 55 : 400-409.

110. Nowak DA, Grefkes C, Ameli M, et al. Interhemispheric competition after stroke: brain stimulation to enhance recovery of function of the affected hand. Neurorehabil Neural Repair 2009; 23: 641-56.

111. Husain M, Shapiro K, Martin J, et al. Abnormal temporal dynamics of visual attention in spatial neglect patients. Nature 1997; 385: 154-156.

112. Robertson IANH, Manly TOM, Beschin N, et al. Auditory sustained attention is a marker of unilateral spatial neglect. Neuropsycologia 1997; 35: 1527-1532.

113. Cotoi A, Mirkowski M, Iruthayarajah J, et al. The effect of theta-burst stimulation on unilateral spatial neglect following stroke: a systematic review. Clin Rehabil 2018; 026921551880401. 
Table 1. Clinical and demographical features of patients included in the studies reviewed.

\begin{tabular}{|c|c|c|c|c|c|c|c|}
\hline Author, year & NIBS & Stroke / TBI & $\mathrm{N}$ of patients & Severity of lesion & Age, y & Duration, $\mathrm{d} / \mathrm{w} / \mathrm{m} / \mathrm{y}$ & $\begin{array}{l}\text { Concomitant } \\
\text { treatments }\end{array}$ \\
\hline \multicolumn{8}{|c|}{ UNCONTROLLED STUDIES } \\
\hline Olivieri, 2001 (31) & rTMS & Stroke & 7 & N/A & between 54-79 & $1-48 w$ & $\mathrm{~N} / \mathrm{A}$ \\
\hline Brighina, 2003 (33) & rTMS & Stroke & 3 & $\mathrm{~N} / \mathrm{A}$ & between $46-67$ & $4-6 m$ & $\mathrm{~N} / \mathrm{A}$ \\
\hline Shindo,2006 (22) & rTMS & Stroke & 2 & $\mathrm{~N} / \mathrm{A}$ & between: $59-61$ & $6 \mathrm{~m}$ & $\begin{array}{l}\text { OT, PT } 5 \text { times/ } \\
\text { week without } \\
\text { special adaptations } \\
\text { or treatment for } \\
\text { USN. }\end{array}$ \\
\hline Bonni, 2013 (19) & rTMS & TBI & 1 & $\mathrm{~N} / \mathrm{A}$ & 20 & $2 y$ & $\mathrm{~N} / \mathrm{A}$ \\
\hline Cao, 2016 (37) & rTMS & Stroke & 10 & $\mathrm{~N} / \mathrm{A}$ & mean: $55 \pm 12$ & $32 \pm 17 d$ & $\mathrm{~N} / \mathrm{A}$ \\
\hline $\begin{array}{l}\text { Johannsen, } 2003 \\
\text { (27) }\end{array}$ & NMV & Stroke & 6 & $\mathrm{~N} / \mathrm{A}$ & between 54-77 & $19-159 \mathrm{~d}$ & CT: PT, OT, NPT. \\
\hline Pitteri, 2013 & NMV & Stroke & 1 & Severe & 43 & $63 d$ & CT \\
\hline Ceyte, 2018 (20) & NMV & Stroke & 1 & NIHSS. 17 & 53 & $18 \mathrm{~m}$ & $\mathrm{~N} / \mathrm{A}$ \\
\hline \multicolumn{8}{|c|}{ CONTROLLED STUDIES } \\
\hline Song, 2009 (32)) & rTMS & Stroke & 14 & $\mathrm{~N} / \mathrm{A}$ & between $43-80$ & $15-60 d$ & $\mathrm{~N} / \mathrm{A}$ \\
\hline Nyffeler, 2009 (30) & rTMS & Stroke & 11 & moderate to severe & $\begin{array}{l}\text { between } \\
40-69\end{array}$ & $4-36 \mathrm{~m}$ & $\mathrm{~N} / \mathrm{A}$ \\
\hline Lim, 2010 (23) & rTMS & Stroke & 7 & $\mathrm{~N} / \mathrm{A}$ & mean: $68 \pm 11.4$ & $9-330 d$ & OT \\
\hline Koch 2012 & rTMS & Stroke & 20 & $\mathrm{~N} / \mathrm{A}$ & $\mathrm{N} / \mathrm{A}$ & $\mathrm{N} / \mathrm{A}$ & $\mathrm{CT} / \mathrm{OT}$ \\
\hline Cazzoli, 2012 (24) & rTMS & Stroke & 24 & $\mathrm{~N} / \mathrm{A}$ & mean: $58 \pm 2.2$ & $26 \pm 4.4 \mathrm{~d}$ & OT, NPT, PT \\
\hline Kim, $2013(21)$ & rTMS & Stroke & 27 & $\mathrm{~N} / \mathrm{A}$ & mean $68 \pm 14$ & $14 \mathrm{~d}$ & CT, PT, OT, \\
\hline
\end{tabular}




\begin{tabular}{|c|c|c|c|c|c|c|c|}
\hline & & & & & & & cognitive therapies \\
\hline Agosta, 2014 & rTMS & Stroke & 6 & $\mathrm{~N} / \mathrm{A}$ & between $51-78$ & $8-33 m$ & $\mathrm{~N} / \mathrm{A}$ \\
\hline Hopfner, 2015 & rTMS & Stroke & 18 & N/A & mean: $62 \pm 10.6$ & $18-63 d$ & $\begin{array}{l}\text { Smooth pursuit eye } \\
\text { movement training }\end{array}$ \\
\hline Fu, 2015 (36) & rTMS & Stroke & 22 & $\mathrm{~N} / \mathrm{A}$ & between :37-78 & $17-114 d$ & CT \\
\hline Cha, 2015 (34) & rTMS & Stroke & 30 & $\mathrm{~N} / \mathrm{A}$ & mean: $64 \pm 12.1$ & $4 \pm 1 \mathrm{~m}$ & $\mathrm{~N} / \mathrm{A}$ \\
\hline Ko, 2008 (29) & tDCS & Stroke & 15 & Moderate to severe & between :44-74 & $31-83 d$ & $\mathrm{~N} / \mathrm{A}$ \\
\hline Sunwoo, 2013 (38) & tDCS & Stroke & 10 & $\mathrm{~N} / \mathrm{A}$ & mean:62.6 \pm 13.3 & $27.8 \pm 60.4 \mathrm{~m}$ & $\mathrm{~N} / \mathrm{A}$ \\
\hline Brem, 2014 (25) & tDCS & Stroke & 1 & NIHSS 11 & 72 & $26 \mathrm{~d}$ & BT \\
\hline $\begin{array}{l}\text { You Gyoung Yi, } \\
2015 \text { (40) }\end{array}$ & tDCS & Stroke & 32 & $\mathrm{~N} / \mathrm{A}$ & between 39-82 & $\mathrm{N} / \mathrm{A}$ & $\mathrm{N} / \mathrm{A}$ \\
\hline Bang, 2015 (42) & tDCS & Stroke & 6 & $\mathrm{~N} / \mathrm{A}$ & mean: $66.0 \pm 4.2$ & $6.5 \pm 1.6$ & CT \\
\hline Smit, 2015 & tDCS & Stroke & 5 & Severe (BI 10-18) & between $52-76$ & $1-12 y$ & $\mathrm{~N} / \mathrm{A}$ \\
\hline $\begin{array}{l}\text { Bornheim, } 2017 \\
(41)\end{array}$ & tDCS & Stroke & 4 & $\mathrm{~N} / \mathrm{A}$ & between 59-74 & $2 d$ & $\mathrm{~N} / \mathrm{A}$ \\
\hline Karnath, 1993 (43) & NMV & Stroke & 8 & $\mathrm{~N} / \mathrm{A}$ & between 44-84 & $20 d-43 m$ & $\mathrm{~N} / \mathrm{A}$ \\
\hline Karnath, 1995 (44) & NMV & Stroke & 4 & $\mathrm{~N} / \mathrm{A}$ & between 48-61 & $5-115 d$ & TENS \\
\hline $\begin{array}{l}\text { Schindler, } 2002 \\
\text { (26) }\end{array}$ & NMV & Stroke & 20 & $\mathrm{~N} / \mathrm{A}$ & between 24-78 & $2-30 m$ & $\begin{array}{l}\text { Visual exploration } \\
\text { training }\end{array}$ \\
\hline Saevarsson 2010 & NMV & Stroke and brain & 12 & NA & between $47-83$ & $3-57 \mathrm{~m}$ & Prism adaptation \\
\hline
\end{tabular}




\begin{tabular}{|l|l|l|l|l|l|l|}
\hline$(28)$ & & tumor & & & & \\
\hline Kamada, 2011 (45) & NMV & Stroke & 11 & N/A & between 54-79 & 4-17 w \\
\hline
\end{tabular}

n: number of patients; N/A: not available; d:day;w:week; m:month; y:year; rTMS: repetitive transcraneal stimulation; tDCS: transcraneal direct current stimulation; NMV: neuromuscular vibration; USN: unilateral spatial neglect; TBI: traumatic brain injury; NIHSS: National Institute of Healt Stroke Scale; TENS: transcutaneous electrical nerve stimulation; BT: Behavioural therapy; OT: ocupational therapy; CT: conventional treatment ; PT: physiotherapy; NPT: neuropsychological training; BI: Barthel Index. 